\title{
Nonlinear long-wavelength torsional Alfvén waves
}

\author{
S. Vasheghani Farahani ${ }^{1}$, V. M. Nakariakov ${ }^{1,2}$, T. Van Doorsselaere ${ }^{3}$, and E. Verwichte ${ }^{1}$ \\ ${ }^{1}$ Centre for Fusion, Space and Astrophysics, Physics Department, University of Warwick, Coventry CV4 7AL, UK \\ e-mail: s.vasheghani-farahani@warwick.ac.uk \\ 2 Central Astronomical Observatory of the Russian Academy of Sciences at Pulkovo, 196140 St Petersburg, Russia \\ ${ }^{3}$ Centrum voor Plasma-Astrofysica, Mathematics Department, Celestijnenlaan 200B bus 2400, 3001 Leuven, Belgium
}

Received 3 November 2010 / Accepted 23 November 2010

\section{ABSTRACT}

\begin{abstract}
Aims. We investigate the nonlinear phenomena accompanying long-wavelength torsional waves in solar and stellar coronae. Methods. The second order thin flux-tube approximation is used to determine perturbations of a straight untwisted and non-rotating magnetic flux-tube, nonlinearly induced by long-wavelength axisymmetric magnetohydrodynamic waves of small, but finite amplitude.

Results. Propagating torsional waves induce compressible perturbations oscillating with double the frequency of the torsional waves. In contrast with plane shear Alfvén waves, the amplitude of compressible perturbations is independent of the plasma- $\beta$ and is proportional to the torsional wave amplitude squared. Standing torsional waves induce compressible perturbations of two kinds, that grow with the characteristic time inversely proportional to the sound speed, and that oscillate at double the frequency of the inducing torsional wave. The growing density perturbation saturates at the level, inversely proportional to the sound speed.
\end{abstract}

Key words. magnetohydrodynamics (MHD) - waves - Sun: corona - Sun: activity - magnetic fields

\section{Introduction}

Alfvén waves are often considered as the primary candidate for the acceleration of solar (e.g. Cranmer 2009) and stellar (e.g. Charbonneau \& MacGregor 1995; Suzuki 2007) winds and coronal heating (e.g. Ofman 2005). Alfvén waves are also considered in the context of the collimation and confinement of astrophysical jets (Bisnovatyi-Kogan 2007), and in core-collapse supernova explosions (Suzuki et al. 2008). However, observational evidence of Alfveń waves in astrophysical plasmas still remains indirect, e.g. as a possible interpretation of non-thermal broadening of coronal emission lines (e.g. Banerjee et al. 2009) in coronal holes.

One of the key ingredients of the theoretical modelling of Alfvén waves in solar and stellar atmospheres is the concept of the nonlinear cascade. It is needed to explain the transfer of wave energy from the low-frequency injection range (that is around $10 \mathrm{mHz}$ in the solar atmosphere) to the highfrequencydissipation range. In one-dimensional models, the nonlinear cascade is connected with nonlinear generation of higher harmonics, and hence steepening of the waves, causing the onset of nonlinear dissipation or of non-MHD dissipative processes. In a uniform plasma, this process is analytically described by a weakly-nonlinear evolutionary equation, known as the Cohen-Kulsrud equation (Cohen \& Kulsrud 1974). The cubically-nonlinear term in this equation accounts for the nonlinear self-interaction of linearly or elliptically polarised, plane Alfvén waves via the wave-induced perturbation of the local Alfvén speed. These perturbations are often referred to as the nonlinearly-induced compressible motions in Alfvén waves. In contrast with the parallel magnetoacoustic waves (e.g., slow waves in the low- $\beta$ plasma of the solar corona), these perturbations exist even in the zero- $\beta$ regime. Circularly polarised plane Alfvén waves are not subject to this effect.
The compressible flows induced by nonlinear Alfvén waves have been intensively studied in the context of solar wind acceleration (e.g. Ofman \& Davila 1998). A modified Cohen-Kulsrud equation describing spherical Alfvén waves in a stratified atmosphere with a radial magnetic field was derived in Nakariakov et al. (2000b) in application to coronal holes. Analytical results are consistent with numerical MHD modelling performed in Torkelsson \& Boynton (1998). More advanced one-dimensional models for nonlinear Alfvén waves in solar and stellar atmospheres in open-field regions include upflows, super-radial magnetic field geometry, non-adiabatic effects and various dissipation mechanisms (Suzuki 2004; Suzuki \& Inutsuka 2005; Suzuki 2007 , 2008). Secularly growing compressible perturbations, induced by standing Alfvén waves, have been considered in the context of coronal loop hydrostatics by Litwin \& Rosner (1998), and in connection with magnetospheric field-line resonances by Tikhonchuk et al. (1995). Secular compressible effects associated with travelling in opposite directions Alfvén waves were found in Verwichte et al. (1999). All those studies were carried out in terms of a shear Alfvén wave, one-dimensional model.

One-dimensional models mentioned above are based upon the assumption that the waves are plane. For long-period Alfvén waves, with periods of the order of typical time scales of loweratmospheric dynamics, this condition is not fulfilled. For example, for a period of $10 \mathrm{~min}$ and an Alfvén speed of $1 \mathrm{Mm} / \mathrm{s}$, the longitudinal wavelength is $600 \mathrm{Mm}$. In a plane wave, the transverse wavelength should be much larger than the longitudinal wavelength. Hence, for the generation of a plane Alfvén wave of a 10-min period, the wave driver should be of the size exceeding the solar diameter. Also there should be no transverse structuring of the plasma in the Alfvén speed, otherwise the wavefront is distorted, and the wave becomes non-planar (e.g. Botha et al. 2000). Thus, the study of the initial stage of the nonlinear cascade in the corona requires consideration of non-planar Alfvén waves. 
In non-uniform plasma structures, Alfvén waves are situated on magnetic surfaces. In the field-aligned structured coronal plasmas, Alfvén waves can be present in the form of torsional modes (see discussion in Van Doorsselaere et al. 2008a,b). Torsional waves are intensively studied theoretically in the context of coronal heating (e.g. Antolin et al. 2008; Copil et al. 2008; Antolin \& Shibata 2010), coronal seismology (Zaqarashvili \& Murawski 2007; Verth et al. 2010) and particle acceleration in solar flares (Fletcher \& Hudson 2008). There is some indirect evidence of torsional standing modes (Zaqarashvili 2003) and propagating waves (Banerjee et al. 2009) in spectroscopic data, and also in microwave emission (Tapping 1983; Grechnev et al. 2003). The aim of this article is to study compressible perturbations induced by long-wavelength weakly-nonlinear torsional waves, which are essentially non-plane.

\section{Compressible flows induced by torsional waves}

We are interested in torsional waves of wavelength much longer than the diameter of the wave-guiding magnetic flux tube with cylindrical coordinates $(r, \varphi, z)$. In an untwisted and non-rotating tube with the equilibrium magnetic field $B_{z 0}$, mass density $\rho_{0}$, and circular cross-section area $A_{0}$, linear torsional waves are twisting azimuthal motions $v_{\varphi}$ accompanied by the appearance of the azimuthal component of the magnetic field $B_{\varphi}$. At the axis of the flux-tube, both quantities vanish, and hence cannot be described by the first order thin magnetic flux theory of Roberts \& Webb (1978), while they appear in the secondorder thin flux-tube approximation of Zhugzhda (1996). The approximation allows one to describe axisymmetric $(m=0$, where $m$ is the azimuthal wave number) flows of plasma, including sausage, longitudinal and torsional modes (Zhugzhda \& Nakariakov 1999; Vasheghani Farahani et al. 2010) and their interaction. Linear torsional waves are governed by the equations for the quantities $\Omega=v_{\varphi} / r$ and $J=B_{\varphi} / r$, which in the thin flux tube approximation correspond to the vorticity and electric current density, respectively,

$\frac{\partial \Omega}{\partial t}-\frac{B_{z 0}}{4 \pi \rho_{0}} \frac{\partial J}{\partial z}=0$

$\frac{\partial J}{\partial t}-B_{z 0} \frac{\partial \Omega}{\partial z}=0$,

where $r$ is the radial coordinate. Also it is worth mentioning that in the magnetic cylinder, a torsional wave could exist on any cylindrical shell (magnetic surface), having an arbitrary dependence on $r$, provided $v_{\varphi}$ and $B_{\varphi}$ are zero on the axis of the cylinder. In the thin flux tube approximation, those dependencies are approximated by linear functions. Equations (1) and (2) are readily combined in the wave equation,

$\left[\frac{\partial^{2}}{\partial t^{2}}-C_{\mathrm{A}}^{2} \frac{\partial^{2}}{\partial z^{2}}\right] J=0$

where $C_{\mathrm{A}}=B_{z 0} / \sqrt{4 \pi \rho_{0}}$ is the Alfvén speed. In the linear regime, these motions are decoupled from compressible motions. The latter are described by the variables $u$ and $V$, the longitudinal and radial components of the velocity, respectively, $\rho$ the mass density, $B_{z}$ the longitudinal component of the magnetic field, $p$ the gas pressure and $A$ the perturbation of the crosssectional area of the flux tube.

A long-wavelength torsional wave of a finite amplitude induces compressible motions by three forces: the centrifugal force connected with the azimuthal rotation of the plasma, the magnetic tension force caused by the magnetic field curvature, and the ponderomotive force that is connected with the longitudinal gradients of the magnetic pressure perturbation in the torsional wave. The first two forces are absent from the plane wave theory of Alfvén waves and appear because of plasma structuring. These forces can modify the flux-tube cross-sectional area, hence inducing compressible plasma motions in the longitudinal and radial directions. In the second-order thin flux-tube approximation, these effects are taken into account in the transverse force-balance equation. The ponderomotive force causes the nonlinear self-interaction of Alfvén waves in the CohenKulsrud equation formalism.

We consider a weakly-nonlinear torsional wave and restrict our attention to the linear terms of the compressible variables, described by the equations

$$
\begin{aligned}
p+\frac{1}{4 \pi} B_{z 0} B_{z} & -\frac{A_{0} \rho_{0}}{2 \pi} \frac{\partial V}{\partial t}-\frac{A_{0} B_{z 0}}{16 \pi^{2}} \frac{\partial^{2} B_{z}}{\partial z^{2}} \\
& =p_{T}^{\mathrm{ext}}+\frac{A_{0} J^{2}}{8 \pi^{2}}-\frac{A_{0} \rho_{0} \Omega^{2}}{2 \pi},
\end{aligned}
$$

$\rho_{0} \frac{\partial u}{\partial t}+\frac{\partial p}{\partial z}=-\frac{1}{4 \pi} J R^{2} \frac{\partial J}{\partial z}$

where $p_{T}^{\text {ext }}$ is the total pressure in the external medium, and $R=\sqrt{A_{0} / \pi}$ is the flux-tube radius. The nonlinear terms associated with the torsional wave are on the right hand side. In Eq. (5) the term responsible for the ponderomotive force appears after accounting for the higher-order terms in the thin flux-tube expansion.

The compressible variables are expressed through the longitudinal component of the magnetic field perturbation $B_{z}$, with the use of the linear expressions

$$
\frac{\partial \rho}{\partial t}+\rho_{0} \frac{\partial u}{\partial z}+2 \rho_{0} V=0
$$

$\frac{\partial B_{z}}{\partial t}+2 B_{z 0} V=0$

$\frac{\partial p}{\partial t}-C_{\mathrm{s}}^{2} \frac{\partial \rho}{\partial t}=0$

where $C_{\mathrm{s}}$ is the sound speed. Equations (6)-(8) are readily combined in a driven wave equation for the density perturbation,

$$
\begin{array}{r}
\left(C_{\mathrm{s}}^{2}+C_{\mathrm{A}}^{2}\right) \mathcal{D}_{T} \rho+\frac{A_{0}}{4 \pi} \mathcal{D}_{\mathrm{s}} \mathcal{D}_{A} \rho=\frac{\partial^{2} p_{T}^{\mathrm{ext}}}{\partial t^{2}}+\frac{A_{0}}{2 \pi} \frac{\partial^{2}}{\partial t^{2}}\left(\frac{J^{2}}{4 \pi}-\rho_{0} \Omega^{2}\right) \\
+\frac{R^{2} C_{\mathrm{A}}^{2}}{4 \pi} \frac{\partial}{\partial z}\left(J \frac{\partial J}{\partial z}\right)+\frac{A_{0} R^{2}}{16 \pi^{2}} D_{\mathrm{A}} \frac{\partial}{\partial z}\left(J \frac{\partial J}{\partial z}\right),(9)
\end{array}
$$

where

$\mathcal{D}_{T, \mathrm{~s}, \mathrm{~A}}=\frac{\partial^{2}}{\partial t^{2}}-C_{T, \mathrm{~s}, A}^{2} \frac{\partial^{2}}{\partial z^{2}}$, and $C_{T}=\frac{C_{\mathrm{A}}^{2} C_{\mathrm{s}}^{2}}{C_{\mathrm{A}}^{2}+C_{\mathrm{s}}^{2}}$.

The last term on the right hand side of Eq. (9) can be neglected in comparison with the other terms, as it is proportional to $A_{0} / \lambda^{2} \ll 1$, where $\lambda$ is the longitudinal wavelength. The second term on the left hand side, responsible for wave dispersion, can be neglected too. Equation (9) describes the excitation 
of compressible motions by weakly nonlinear, long-wavelength torsional waves in a thin magnetic flux tube. In the following we ignore the perturbation of the total pressure in the external medium, $p_{T}^{\text {ext }}$, concentrating on the compressible flows inside the flux-tube.

$$
\begin{aligned}
\left(C_{\mathrm{s}}^{2}+C_{\mathrm{A}}^{2}\right) D_{T} \rho= & \frac{A_{0}}{2 \pi} \frac{\partial^{2}}{\partial t^{2}}\left(\frac{J^{2}}{4 \pi}-\rho_{0} \Omega^{2}\right) \\
& +\frac{R^{2} C_{\mathrm{A}}^{2}}{4 \pi} \frac{\partial}{\partial z}\left(J \frac{\partial J}{\partial z}\right),
\end{aligned}
$$

also, the back-reaction of the induced compressible flows on the torsional waves through the modification of the local Alfvén speed and the flux-tube diameter is not considered. The latter assumption is justified by the consideration of the quadratically nonlinear terms only, while the consideration of the Alfvén wave self-interaction appears when the cubic nonlinearity is taken into account.

The first term on the right hand side of Eq. (11) has two terms associated with the nonlinear torsional wave, which have opposite signs. Hence, their combined effect on the compressible flows depends upon the phase relations between the twist and the rotation of the plasma in the torsional waves. Consider separately the cases of propagating and standing waves.

\section{Propagating torsional waves}

We take a propagating solution of Eq. (11),

$J=j_{\mathrm{a}} \cos (\omega t-k z)$,

where $j_{\mathrm{a}}$ is the amplitude of the magnetic twist, and $\omega$ and $k$ are the frequency and the wavenumber, respectively, for which $\omega= \pm C_{\mathrm{A}} k$. We use Eq. (1) to express the associated rotation of the flux-tube as

$\Omega=-\frac{j_{\mathrm{a}}}{\left(4 \pi \rho_{0}\right)^{1 / 2}} \cos (\omega t-k z)$

For such a solution, the first term on the right-hand side of Eq. (11) vanishes:

$$
\frac{J^{2}}{4 \pi}-\rho_{0} \Omega^{2}=\left(\frac{j_{\mathrm{a}}^{2}}{4 \pi}-\rho_{0} \frac{\left(-j_{a}\right)^{2}}{4 \pi \rho_{0}}\right) \cos ^{2}(\omega t-k z)=0 .
$$

This means that the effects of nonlinear magnetic twist and plasma rotation in the travelling wave cancel out each other, and do not add new effects to the nonlinear compressibility of propagating torsional waves. Thus, the induced compressible motions are described by the equation

$\left(C_{\mathrm{s}}^{2}+C_{\mathrm{A}}^{2}\right) \mathcal{D}_{T} \rho=-\frac{R^{2} C_{\mathrm{A}}^{2}}{4 \pi} k^{2} j_{\mathrm{a}}^{2} \cos [2(\omega t-k z)]$.

with the driven solution

$\rho=\frac{R^{2} j_{\mathrm{a}}^{2}}{16 \pi C_{\mathrm{A}}^{2}} \cos [2(\omega t-k z)]$.

Thus, we obtain that the right hand side of Eq. (16) is independent of the value of the sound speed.

Compare Eq. (16) with the case without transverse structuring, i.e. with plane shear Alfvén waves. Consider waves propagating in the $z$-direction, taking $\partial / \partial y=0$ and $\partial / \partial x=0$. Restrict our attention to the linearly polarised Alfvén waves, $v_{y}$ and $B_{y}$.
Following the formalism developed in Nakariakov et al. (2000a), we obtain the equivalent of Eq. (11)

$\mathcal{D}_{\mathrm{s}} \rho=\frac{1}{4 \pi} \frac{\partial}{\partial z} B_{y} \frac{\partial B_{y}}{\partial z}$

Taking $B_{y}=B_{y \mathrm{a}} \cos (\omega t-k z)$ we get

$\mathcal{D}_{\mathrm{s}} \rho=-\frac{1}{4 \pi} B_{y \mathrm{a}}^{2} k^{2} \cos (2 \omega t-2 k z)$,

with the solution

$\rho=\frac{B_{y \mathrm{a}}^{2} k^{2}}{16 \pi\left(\omega^{2}-C_{\mathrm{A}}^{2} k^{2} \beta\right)} \cos (2 \omega t-2 k z)$,

where $\beta=C_{\mathrm{s}}^{2} / C_{\mathrm{A}}^{2}$. With $j_{\mathrm{a}} \approx B_{\varphi a} / R$, we observe that the right hand side terms in Eqs. (16) and (19) are of the similar order. However, there is an important difference between solutions (16) and (19) is that in the case of torsional waves, there is no possibility for a resonance of the Alfvén waves with the sound wave. Note, in the zero- $\beta$ limit, both solutions coincide.

\section{Standing torsional waves}

We consider standing torsional waves that may appear in closed magnetic structures, e.g. coronal loops with $k=\pi / L$ where $L$ is the loop length

$J=2 j_{a} \cos (\omega t) \cos (k z)$,

$\Omega=-2 \frac{j_{a}}{\left(4 \pi \rho_{0}\right)^{1 / 2}} \sin (\omega t) \sin (k z)$,

where the phase relations between the perturbed magnetic twist and the vorticity are obtained from Eqs. (1) and (2). Substituting Eqs. (20) in Eq. (11), and neglecting dispersive effects, higher order terms and the perturbations of the external medium, we obtain

$$
\begin{aligned}
\left(C_{\mathrm{s}}^{2}+C_{\mathrm{A}}^{2}\right) \mathcal{D}_{T} \rho= & -\frac{R^{2} C_{\mathrm{A}}^{2}}{2 \pi} j_{a}^{2} k^{2}(1+\cos (2 \omega t)) \cos (2 k z) \\
& -\frac{A_{0} j_{a}^{2} \omega^{2}}{\pi^{2}} \cos (2 \omega t) .
\end{aligned}
$$

The first term on the right hand side represents the ponderomotive effect, and the second term contains the magnetic tension and centrifugal effects.

In the finite- $\beta$ case with the constraint $\beta \ll 1$ the solution for Eq. (21) is

$$
\begin{aligned}
\rho= & -\frac{R^{2} j_{a}^{2}}{8 \pi C_{s}^{2}(1+\beta)}\left(1-\cos \left(2 C_{s} k t\right)\right) \cos (2 k z) \\
& +\left(\frac{R^{2}}{4 \pi C_{\mathrm{A}}^{2}(1+\beta)}+\frac{R^{2} \cos (2 k z)}{8 \pi C_{\mathrm{A}}^{2}}\right) \times j_{a}^{2} \cos (2 \omega t),
\end{aligned}
$$

where $\omega=C_{\mathrm{A}} k$. Equation (22) is similar to Eq. (13) of Tikhonchuk et al. (1995) obtained for shear Alfvén waves.

According to Eq. (22), standing torsional waves induce growing compressible perturbations, similarly to standing shear Alfvén waves (Tikhonchuk et al. 1995; Verwichte et al. 1999; Litwin \& Rosner 1998) and standing kink modes of coronal loops (Terradas \& Ofman 2004). The growth is connected with the ponderomotive term on the right hand side of Eq. (21). 
Finite- $\beta$ effects cause saturation of the compressible perturbation. Using the results obtained for shear Alfvén waves, we obtain that the highest value of the density perturbation,

$\frac{\rho}{\rho_{0}}=\frac{R^{2} j_{a}^{2}}{4 \pi \rho_{0}} \frac{1}{C_{\mathrm{s}}^{2}\left(1+C_{\mathrm{s}}^{2} / C_{A}^{2}\right)}$,

is reached at the time $t_{\max } \simeq L / 2 C_{\mathrm{s}}$, where $L$ is the loop length.

Also, in the case of standing waves, the centrifugal and magnetic tension terms do not cancel each other, and produce compressible perturbations oscillating at the double frequency of the torsional wave. However, those terms do not cause the secular growth of compressible perturbations.

\section{Conclusions}

We considered compressible perturbations induced in straight untwisted and non-rotating magnetic flux tubes by weaklynonlinear long-wavelength torsional waves. We can summarise our findings as follows:

1. Long-wavelength torsional waves induce compressible perturbations by the ponderomotive, centrifugal and magnetic twist forces. The perturbations have double the frequency of the inducing torsional wave. The efficiency of the excitation depends upon the spatial (standing and propagating) structure of the inducing torsional wave.

2. The efficiency of the generation of compressible perturbations by long-wavelength torsional waves is independent of the plasma- $\beta$ (see Eq. (16)). This result is different from the excitation of compressible perturbations by plane shear Alfvén waves, in which case the efficiency grows when the Alfvén and sound speeds approach each other. The discrepancy is connected with the fact that the tube speed is always lower than the Alfvén speed. The relative amplitude of the induced density perturbation is $\rho / \rho_{0}=B_{\varphi}^{2} / 4 B_{z 0}^{2}$, where $B_{\varphi}$ is the perturbation of the magnetic field at the boundary of the flux tube.

3. There are two kinds of compressible perturbations induced by standing torsional waves: the perturbations which grow with the time scale $1 / 2 C_{\mathrm{s}} k$, where $k$ is the longitudinal wave number of the torsional wave, and the perturbations oscillating at the double frequency of the driving torsional mode. The growing density perturbations saturate at the level inversely proportional to the sound speed.

Thus we conclude that nonlinear compressible effects which accompany standing weakly-nonlinear long-wavelength torsional waves are similar to those derived for plane shear Alfvén waves. For propagating waves, the efficiency of the nonlinear generation of compressible perturbations does not grow with the plasma beta. This effect should be taken into account in one-dimensional models of the solar and stellar wind acceleration by Alfvén waves.

Acknowledgements. T.V.D. is a post-doctoral researcher of the FWO Vlaanderen.

\section{References}

Antolin, P., \& Shibata, K. 2010, ApJ, 712, 494

Antolin, P., Shibata, K., Kudoh, T., Shiota, D., \& Brooks, D. 2008, ApJ, 688, 669

Banerjee, D., Pérez-Suárez, D., \& Doyle, J. G. 2009, A\&A, 501, L15

Bisnovatyi-Kogan, G. S. 2007, MNRAS, 376, 457

Botha, G. J. J., Arber, T. D., Nakariakov, V. M., \& Keenan, F. P. 2000, A\&A, 363, 1186

Charbonneau, P., \& MacGregor, K. B. 1995, ApJ, 454, 901

Cohen, R. H., \& Kulsrud, R. M. 1974, Phys. Fluids, 17, 2215

Copil, P., Voitenko, Y., \& Goossens, M. 2008, A\&A, 478, 921

Cranmer, S. R. 2009, Living Rev. Sol. Phys., 6, 3

Fletcher, L., \& Hudson, H. S. 2008, ApJ, 675, 1645

Grechnev, V. V., White, S. M., \& Kundu, M. R. 2003, ApJ, 588, 1163

Litwin, C., \& Rosner, R. 1998, ApJ, 506, L143

Nakariakov, V. M., Mendoza-Briceño, C. A., \& Ibáñez S., M. H. 2000a, ApJ, 528,767

Nakariakov, V. M., Ofman, L., \& Arber, T. D. 2000b, A\&A, 353, 741

Ofman, L. 2005, Space Sci. Rev., 120, 67

Ofman, L., \& Davila, J. M. 1998, J. Geophys. Res., 103, 23677

Roberts, B., \& Webb, A. R. 1978, Sol. Phys., 56, 5

Suzuki, T. K. 2004, MNRAS, 349, 1227

Suzuki, T. K. 2007, ApJ, 659, 1592

Suzuki, T. K. 2008, Nonlinear Proc. Geophys., 15, 295

Suzuki, T. K., \& Inutsuka, S. 2005, ApJ, 632, L49

Suzuki, T. K., Sumiyoshi, K., \& Yamada, S. 2008, ApJ, 678, 1200

Tapping, K. F. 1983, Sol. Phys., 87, 177

Terradas, J., \& Ofman, L. 2004, ApJ, 610, 523

Tikhonchuk, V. T., Rankin, R., Frycz, P., \& Samson, J. C. 1995, Phys. Plasmas, 2, 501

Torkelsson, U., \& Boynton, G. C. 1998, MNRAS, 295, 55

Van Doorsselaere, T., Brady, C. S., Verwichte, E., \& Nakariakov, V. M. 2008a, A\&A, 491, L9

Van Doorsselaere, T., Nakariakov, V. M., \& Verwichte, E. 2008b, ApJ, 676, L73

Vasheghani Farahani, S., Nakariakov, V. M., \& Van Doorsselaere, T. 2010, A\&A, 517, A29

Verth, G., Erdélyi, R., \& Goossens, M. 2010, ApJ, 714, 1637

Verwichte, E., Nakariakov, V. M., \& Longbottom, A. W. 1999, J. Plasma Phys., 62,219

Zaqarashvili, T. V. 2003, A\&A, 399, L15

Zaqarashvili, T. V., \& Murawski, K. 2007, A\&A, 470, 353

Zhugzhda, Y. D. 1996, Phys. Plasmas, 3, 10

Zhugzhda, Y. D., \& Nakariakov, V. M. 1999, Phys. Lett. A, 252, 222 